\title{
Parenteral Nutrition in Patients with Advanced Cancer and Gastrointestinal Failure or Obstruction
}

\author{
Irit Chermesh ${ }^{1}$, Nissim Haim² ${ }^{2}$ Amnon Amit ${ }^{3}$, Matti Waterman ${ }^{1}$, Irina Papier ${ }^{4} \&$ Tania Mashiach $^{5}$ \\ ${ }^{1}$ Gastroenterology Department, Rambam Health Care Campus, Israel \\ ${ }^{2}$ Oncology Institute, Rambam Health Care Campus, Israel \\ ${ }^{3}$ Obstetrics and Gynecology, Rambam Health Care Campus, Israel \\ ${ }^{4}$ Nursing Management, Rambam Health Care Campus, Israel \\ ${ }^{5}$ Quality Assurance, Rambam Health Care Campus, Israel \\ Correspondence: Irit Chermesh, Gastroenterology Department, Rambam Health Care Campus, Israel. E-mail: \\ I_chermesh@rambam.health.gov.il
}

Received: July 19, 2016

doi:10.5539/cco.v5n2p54
Accepted: August 30, $2016 \quad$ Online Published: October 27, 2016

URL: http://dx.doi.org/10.5539/cco.v5n2p54

\begin{abstract}
Malnutrition in patients with advanced cancer and gastrointestinal failure (ACGIF) is inevitable. This is a prospective study of a cohort of patients with ACGIF who were treated by home parenteral nutrition (HPN).

Included were all patients with ACGIF treated with HPN since January 2003. Data concerning the primary disease, demographics, functional capacity, and PN-related complications were obtained.

125 patients, age's $60 \pm 12.9$ years, were included. Seven $(6 \%)$ had KPS $<50$. Median survival was 3.2 months. Cumulative survival was $0.95,0.50,0.25$, and 0.09 at two weeks, three and six months, and one year after initiation of HPN, respectively. Multivariate analysis revealed higher mortality associated with male gender (HR 1.48 95\% CI 1.03-2.14; $\mathrm{p}<0.05$ ), albumin level<2.5 gr\% (HR 1.73 95\% CI 1.18-2.54 p<0.05), and low functional capacity (KPS<50) (HR $2.6395 \%$ CI 1.21-5.74; p<0.05). Survival was not associated with age or BMI.

More than $50 \%$ of patients survived more than three months, and more than $95 \%$ survived longer than two weeks. We cautiously suggest that HPN has prolonged survival and prevented starvation-related manifestations. The decision to initiate HTPN must be reached based on individual considerations, taking into account patient's preferences.
\end{abstract}

Keywords: parenteral nutrition, home parenteral nutrition, palliative care, advanced cancer, performance status, malnutrition

\section{Background}

Cancer induced weight loss in advanced cancer patients is a complex multifactorial phenomenon. Malnutrition due to reduced intake and/or maldigestion and/or malabsorption and/or stomal losses combined with metabolic derangements lead to disease-related cachexia. Gastrointestinal failure can evolve as a result of obstruction or partial obstruction of the gastrointestinal tract; radiation-induced enteropathy, entero-enteral and enterocutaneous fistulas; chemotherapy-induced side effects; or ileus due to malignant involvement of the mesenteric plexus. Patients with intestinal failure cannot keep their food intake to match their nutritional needs, which inevitably leads to malnutrition. In this group of advanced cancer patients, a question arises regarding the relevance of nutrition in the face of an incurable disease. There are no studies that assess survival in situations of starvation, probably because such studies cannot be ethically performed. Present knowledge is derived from historic events such as the siege of Leningrad, Jewish ghettos during World War Two, and hunger strikes (Brozek et al., 1946; Fliederbaum, 1979; Winick, 1979; Kirbas et al., 2008). Healthy people can survive approximately two and a half months without food intake, although various clinical and neuropsychiatric manifestations of starvation appear as early as 2-3 weeks into the process (Kirbas et al., 2008). If the only nutrition provided is parenteral and if survival exceeds two weeks of TPN, it may be possible to infer that total parenteral nutrition (TPN) played a role in the patient's wellbeing; if survival exceeds two months, it may be possible to infer that TPN played a role in prolonging survival. 
The current study aimed to assess the survival and HPN-related complications in advanced cancer patients with GI failure treated by HTPN. This study is an extension of a previously published series of 28 patients (6).

\section{Methods}

This was a prospective analysis performed in a single tertiary care center in Northern Israel and included all patients with advanced cancer and GI failure, who were considered for HTPN by the attending physicians. All patients were co-assessed by a specialized multi-disciplinary nutrition team. Individual recommendations were made taking into account nutritional status, functional status assessed by the Karnofsky Performance Status (KPS) scale, and life expectancy. The options were discussed with the patients and their families. HTPN was administered until death or near death, as judged by the attending physician, and the decision was made jointly with the patients and their families. All patients were followed at our outpatient clinic. Follow-up was performed using clinical assessment as well as laboratory assessment which were custom-tailored for each patient. All adult patients, 18 years or older, suffering from advanced cancer and GI failure, treated by HTPN and discharged since January 2003 untill January 2015 were included in the analysis. Demographic data, baseline BMI, serum albumin level, weight loss, disease-related data, data regarding HTPN complications, and survival were recorded.

Subgroups were defined and statistical analysis performed accordingly. Three BMI groups were defined based on WHO definition of underweight (BMI $<18)$, normal range (18-25), and overweight (MBI $>25)$.

The study protocol was approved by the institutional review board.

Multivariate analysis of survival was performed using Cox regression. Hazard ratios (HR), with $95 \%$ confidence intervals (CI) and p values, were calculated for factors of overall survival (OS). Kaplan-Meier survival curves were used to illustrate the main results. Two-tailed $\mathrm{p}$ values of 0.05 or less were considered to be statistically significant. Statistical analysis was performed using SPSS 21.0 software for Windows.

\section{Results}

The main characteristics of 125 patients, and multivariate analysis or patient mortality are shown in Table 1 . Fifty five patients (44\%) were male. Age at HPN initiation ranged between 30-93 years, with a median age of 60 \pm 12.9 years. The main diagnosis of primary malignancy included upper GI in 47 patients (38\%), ovarian cancer in $22(18 \%)$, colorectal carcinoma in $18(14 \%)$, pancreatic cancer in $13(10 \%)$, and others $25(20 \%)$. BMI was less than 18.5 in 45 patients (36\%), and weight loss of $>10 \%$ was recorded in 74 patients (59\%). Serum albumin was $<2.5 \mathrm{gr} / \mathrm{dl}$ in more than half the patients.

Overall, 14,848 days of TPN were administered, an average of 119 \pm 118 (median 113) days per patient (range of 8-752). Nighty six (77\%) did not experience any serious complication; $23(18 \%)$ had a single complication and 6 (5\%) experienced 2 complications or more. The most frequent complication was infection related to the central venous catheter, which was diagnosed in 16 patients (12.8\%). In 8 of these, it was associated with sepsis and in the remaining 8 it was local. Other complications included technical problems with the line (5), electrolyte disturbances (2), dehydration (2), and others (3). Overall, 37 parenteral nutrition-related complications were recorded, adding up to 0.24 complications and 0.23 hospitalizations per 100 treatment days. There were no HTPN-related deaths. Cumulative survival was 0.95, 0.50, 0.25, and 0.09 at two weeks, three months, six months, and one year after initiation of HTPN, respectively (figure 1). At the time of analysis, eight patients (6.4\%) remained alive on HTPN. Overall median survival period was 3.2 months. HTPN was stopped when imminent death was perceived. Multivariate analysis found higher mortality associated with male gender (HR 1.48 95\% CI 1.03-2.14; p<0.05), albumin level<2.5 gr\% (HR 1.73 95\% CI 1.18-2.54 p<0.05), and poor functional capacity (KPS<50) (HR 2.63 95\% CI 1.21-5.74; $\mathrm{p}<0.05$ ). Patients who lost $10 \%$ or more of their body weight had lower survival rates (HR 0.54 CI $95 \% 0.33-0.88$; $\mathrm{p}<0.05$ ) than those who lost less than $10 \%$. There was no difference in survival associated with age or BMI. 
Table 1. Characteristics of 125 patients treated with HTPN, and analysis of patient mortality

\begin{tabular}{|c|c|c|c|c|c|c|c|c|c|c|}
\hline \multicolumn{11}{|c|}{ Overall mortality } \\
\hline & & & 2 weeks & 3 months & 6months & 1 year & & & $95 \% \mathrm{CI}$ & \\
\hline & & Pts. $^{a}$ & No. ${ }^{b}(\%)$ & No. & No. $(\%)$ & No. $(\%)$ & P value & $\mathbf{H R}^{\mathrm{c}}$ & Lower & Upper \\
\hline \multirow{3}{*}{ Gender } & Total & $125(\%)$ & $6(4.8)$ & $62(49.6)$ & $86(68.8)$ & $104(83.2)$ & & & & \\
\hline & $\mathrm{F}$ & $70(56)$ & $1(1.4)$ & $29(41.4)$ & $47(67.1)$ & $58(82.9)$ & & 1.00 & & \\
\hline & M & $55(44)$ & $5(9.1)$ & $33(60.0)$ & $39(70.9)$ & $46(83.6)$ & 0.046 & 1.45 & 1.01 & 2.10 \\
\hline \multirow[t]{3}{*}{ Age } & $<50$ & $25(20)$ & 0 & $15(60.0)$ & $17(68.0)$ & $20(80.0)$ & 0.780 & 1.00 & 0.00 & 0.00 \\
\hline & $50-64$ & $59(47)$ & $4(6.8)$ & $27(45.8)$ & $39(66.1)$ & $50(84.7)$ & 0.482 & 1.19 & 0.73 & 1.96 \\
\hline & $65+$ & 41(33) & $2(4.9)$ & $20(48.8)$ & $30(73.2)$ & 34 (82.9) & 0.609 & 1.15 & 0.68 & 1.95 \\
\hline \multirow[t]{2}{*}{$\mathbf{K P S}^{\mathbf{d}}$} & $50+$ & $114(91)$ & $5(4.4)$ & $54(47.4)$ & $76(66.7)$ & $94(82.5)$ & & 1.00 & & \\
\hline & $<50$ & $7(6)$ & 0 & $5(71.4)$ & $7(100)$ & $7(100.0)$ & 0.015 & 2.63 & 1.21 & 5.74 \\
\hline \multirow[t]{4}{*}{$\mathbf{B M I}^{\mathrm{e}}$} & $<18.5$ & $45(36)$ & $2(4.4)$ & $19(42.2)$ & $29(64.4)$ & $34(75.6)$ & 0.354 & 1.00 & 0.00 & 0.00 \\
\hline & $18.5-24.9$ & $65(52)$ & $2(3.1)$ & $35(53.8)$ & $48(73.8)$ & $58(89.2)$ & 0.181 & 1.32 & 0.88 & 1.97 \\
\hline & $25+$ & $14(11)$ & $1(7.1)$ & $7(50.0)$ & $9(64.3)$ & $12(85.7)$ & 0.307 & 1.39 & 0.74 & 2.62 \\
\hline & Missing & $1(1)$ & $1(100)$ & $1(100)$ & 0 & 0 & & & & \\
\hline \multirow[t]{4}{*}{ Weight loss } & $<5 \%$ & 24 (19) & $3(12.5)$ & $14(58.3)$ & $18(75)$ & $20(83.3)$ & 0.045 & 1.00 & 0.00 & 0.00 \\
\hline & $5-9 \%$ & $19(15)$ & $1(5.3)$ & $10(52.6)$ & $12(63.2)$ & $16(84.2)$ & 0.107 & 0.59 & 0.31 & 1.12 \\
\hline & $10 \%+$ & $74(59)$ & $2(2.7)$ & $31(41.9)$ & $49(66.2)$ & $60(81.1)$ & 0.013 & 0.54 & 0.33 & 0.88 \\
\hline & Missing & $8(6)$ & 0 & $7(87.5)$ & $7(87.5)$ & $8(100)$ & & & & \\
\hline \multirow[t]{7}{*}{ Diagnosis } & $\mathrm{CRC}$ & $18(14)$ & 0 & 7 (38.9) & $10(55.6)$ & $14(77.8)$ & 0.161 & 1.00 & 0.00 & 0.00 \\
\hline & Breast & $6(5)$ & 0 & $2(33.3)$ & $3(50.0)$ & $4(66.7)$ & 0.674 & 0.80 & 0.29 & 2.22 \\
\hline & Upper GI & $47(38)$ & $2(4.3)$ & $24(51.1)$ & $34(72.3)$ & $41(87.2)$ & 0.318 & 1.35 & 0.75 & 2.43 \\
\hline & Head and neck & $8(6)$ & $2(25.0)$ & $5(62.5)$ & $5(62.5)$ & $6(75.0)$ & 0.026 & 2.66 & 1.12 & 6.32 \\
\hline & Ovary & $22(22)$ & $2(9.1)$ & $15(68.2)$ & $17(77.3)$ & $18(81.8)$ & 0.143 & 1.65 & 0.84 & 3.24 \\
\hline & Pancreas & $13(10)$ & 0 & $6(46.2)$ & $8(61.5)$ & $11(84.6)$ & 0.941 & 0.97 & 0.45 & 2.09 \\
\hline & Other & $11(9)$ & 0 & $3(27.3)$ & $9(81.8)$ & $10(90.9)$ & 0.950 & 0.98 & 0.44 & 2.17 \\
\hline \multirow[t]{3}{*}{ Albumin } & $\geq 2.5$ & $54(43)$ & $1(1.9)$ & $24(44.4)$ & $33(61.1)$ & $44(81.5)$ & & 1.00 & & \\
\hline & $<2.5$ & $68(54)$ & $4(5.9)$ & $35(51.5)$ & $51(75.0)$ & $58(85.3)$ & 0.005 & 1.73 & 1.18 & 2.54 \\
\hline & Not available & 3 & $1(33.3)$ & 3 & $2(66.7)$ & $2(66.7)$ & & & & \\
\hline
\end{tabular}
a. Pts. - patients
b. No.- number
c. HR- Hazard Ratio
d. KPS- karnofsky performance scale
e. BMI- body mass index

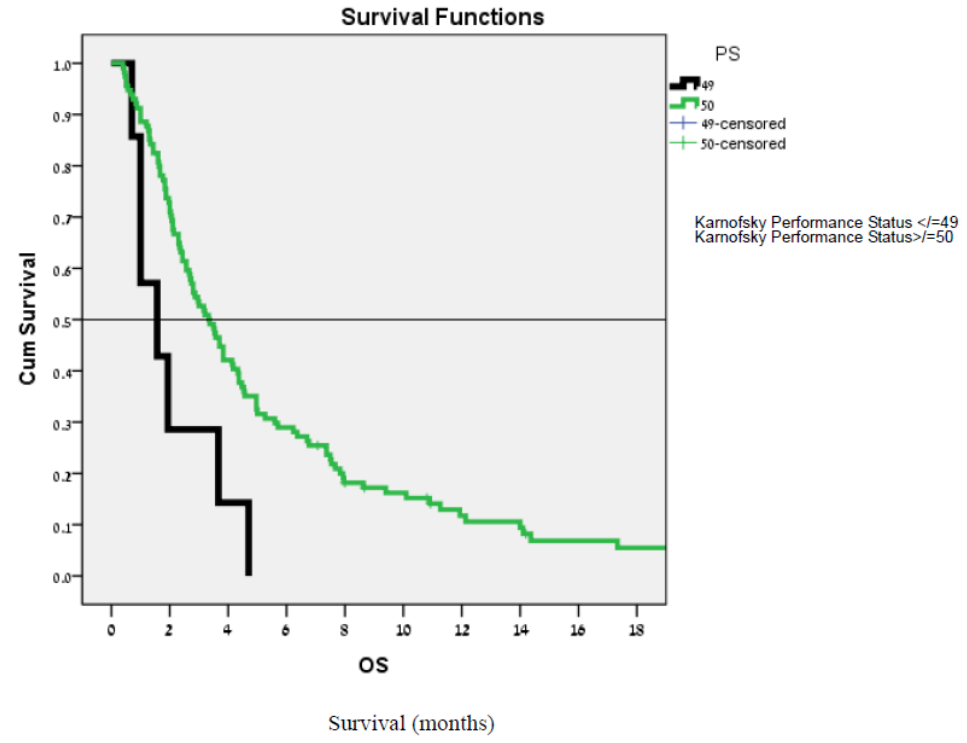

Figure 1. Overall survival and performance status

\section{Discussion}

This is the largest published cohort of patients with advanced cancer and GI failure treated with HTPN. The main goals of the HTPN treatment were prolonging life and improving quality of life.

This was a prospective observational study. In view of two goals of the treatment, it is highly unlikely that a double-blind controlled study could be conducted to assess the survival of patients with and without nutrition 
when the enteral route is no longer available. Oh et al. conducted a study in which patients were randomized to receive either TPN or parenteral fluid (Oh et al., 2014), but it was interrupted because patients and families were concerned about starvation. Relying on knowledge from other fields and use common sense, logic, and compassion is advocated. It is known that humans present clinical manifestations of starvation after about two weeks of food deprivation, and that survival is limited to about two months of total starvation (Leiter et al., 1982) A logical hypothesis is that if survival of patients receiving TPN exceeds two weeks, they are spared the clinical manifestations of starvation, and if survival exceeds two months, nutrition plays a role in prolonging survival. According to the guidelines of the Fédération Nationale des Centres de Lutte Contre le Cancer (FNCLCC), parenteral nutrition in a palliative setting can slow down nutritional deprivation, help avoid dehydration, and improve quality of life in patients with a malignant bowel obstruction and in other cases of food intolerance (Bachmann et al., 2003). Overall, relatively high survival rates were observed at 2 weeks and at 3 months in the current study, including in poor risk subgroups (low KPS, low albumin, and male gender).

Providing optimal treatment to patients with advanced cancer involves complex weighing of the advantages and disadvantages of treatment options. Loss of weight in patients with advanced cancer is multifactorial with malnutrition and cancer cachexia each playing a role. Cancer cachexia as a paraneoplastic syndrome is caused by complex interactions between inflammation, hypermetabolism (increased resting energy expenditure, catabolism of muscle protein), neuro-hormonal changes and proteolytic and lipolytic factors produced by the host and the tumor (Blum et al., 2010; Blum et al., 2010). Cancer Cachexia is not reversed with nutritional treatment. There are many factors associated with malnutrition contributing to this outcome. Reduced intake of nutrition plays a significant role in some of the patients. Patients suffering from GI failure by definition cannot ingest enough food to supply their needs, but even among these patients it is necessary to determine which patients benefit the most of HTPN thus outweighing the risks, the inconvenience and the cost. Recommendations of HTPN for advanced cancer patients with GI failure are based on assessment of nutrient intake ability and survival expectancy. Naghibi et al published a review and meta-analysis of 12 studies involving 437 patients with inoperable malignant bowel obstruction (Naghibi et al., 2014). Mean survival was 116 days; the median was 83 days, with $45 \%$ still alive at 3 months and $24 \%$ at 6 months, but only $2 \%$ at one year. These numbers are somewhat worse than the survival recorded in our study. According to the expert opinion of the FNCLCC, there is no justification for PN in patients with a KPS of $50 \%$ or lower (Bachmann et al., 2003). According to the guidelines of the European Society for Clinical Nutrition and Metabolism (ESPEN), TPN should be considered if survival is estimated to be longer than 2-3 months (Bozzetti et al., 2009) but no tool is available to assess survival with any precision. In a study by Pironi et al., survival was underestimated in about a third of patients (Pironi et al. 1997). In a position paper Schneider et al. stress that the ability to estimate the length of survival is rather limited (Schneider et al., 2015). In the cohort hereby described, about 40\% of patients with a KPS score of $<50$ survived more than three months. Patients who lose a considerable precentage of their body weight form a distinct group that might gain considerably from HTPN. It is reasonable to assume that reversible malnutrition plays a significant role in this group of patients, who can therefore gain from HTPN. The data illustrate the complexities involved in selecting patients who might gain from being treated with HTPN. Because of limited ability to estimate, HTPN is provided either to a large group of patients, some of whom surviving only a short period of time and not being able to gain from this treatment, or to a carefully selected group, in which case HTPN may be denied to some patients who could benefit from it.

QoL is the second goal of treatment. Culine and Senesse et al. described cohorts of HTPN patients (767 with various cancers and 370 with GI cancers, respectively) (Culine et al., 2014; Senesse et al. 2015). Vashi et al described a cohort of 52 patients with advanced cancer, treated by TPN (Vashi et al., 2014). The patients in these studies comprise a somewhat a different goup, since there was a considerable proportion, who were under active anti-neoplastic treatment, whereas most of the patients described in the current study were beyond active anti-neoplastic treatment. In these studies QoL improved over the time and the rates of adverse events and treatment-related discomfort were acceptable. With or without hospitalizations, HTPN complications can significantly lower the quality of life. A major drawback of the current study is that QoL was not assessed. Additional limitations are that the data gathered was limmited to the routine follow-up. No data regarding immune function or susseptability to infection was available.

In the complex situations described above, where there is no sharp distinction between those who should and should not receive HTPN, the data should be made available to patients and their families, and decisions reached jointly.

\section{Conclusion}

HTPN can prolong life and prevent starvation-related manifestations in a subgroup of patients with advanced 
cancer and GI failure. This outcome is achieved at the cost of treatment inconvenience and a risk of complications. Decision about HTPN should take into account nutritional status and GI functioning. Life expectancy is also important, but it is important to acknowledge our limited ability to estimate survival benefit from HTPN. We recommend individualized, well-informed, joint decisions with the patients and their families.

\section{Conflict of interest statement}

Authors declare no conflict of interest. There was no funding for this study.

\section{References}

Bachmann, P., Marti-Massoud, C., Blanc-Vincent, M. P., Desport, J. C., Colomb, V., Dieu, L., ... Senesse, P. (2003). Summary version of the Standards, Options and Recommendations for palliative or terminal nutrition in adults with progressive cancer (2001). Br J Cancer, 89(1), 107-10.

http://dx.doi.org/10.1038/sj.bjc.6601092

Blum, D., Omlin, A., Baracos, V. E., Solheim, T. S., Tan, B. H., Stone, P., Kaasa, S., Fearon, K., \& Strasser, F. (2011). European Palliative Care Research Collaborative. Cancer cachexia: a systematic literature review of items and domains associated with involuntary weight loss in cancer. Crit Rev Oncol Hematol, 80(1), 114-44. http://dx.doi.org/10.1016/j.critrevonc.2010.10.004

Blum, D., Omlin, A., Fearon, K., Baracos, V., Radbruch, L., Kaasa, S., \& Strasser, F. (2010). European Palliative Care Research Collaborative. Evolving classification systems for cancer cachexia: ready for clinical practice? Support Care Cancer, 18(3), 273-9. http://dx.doi.org/10.1007/s00520-009-0800-6

Bozzetti, F., Arends, J., Lundholm, K., \& Micklewright, A. (2009). Zurcher G and Muscaritoli M: ESPEN Guidelines on parenteral nutrition: non-surgical oncology. Clin Nutr, 28, 445-454. http://dx.doi.org/10.1016/j.clnu.2009.04.011

Brozek, J., Well, S., \& Keys, A. (1946). Medical aspects of semistarvation in Leningrad siege. Am Rev Sov Med 4, 1941-1942.

Chermesh, I., Mashiach, T., Amit, A., Haim, N., Papier, I., Efergan, R., Lachter, J., \& Eliakim, R. (2011). Home parenteral nutrition (HTPN) for incurable patients with cancer with gastrointestinal obstruction: do the benefits outweigh the risks? Med Oncol., 28(1), 83-8. http://dx.doi.org/10.1007/s12032-010-9426-2

Culine, S., Chambrier, C., Tadmouri, A., Senesse, P., Seys, P., Radji, A., Rotarski, M., Balian, A., \& Dufour, P. (2014). Home parenteral nutrition improves quality of life and nutritional status in patients with cancer: a French observational multicenter study. Support Care Cancer. 22(7), 1867-74. http://dx.doi.org/10.1007/s00520-014-2164-9

Fliederbaum, J. (1979). Clinical aspects of hunger disease in adults. In: Winick M, editor. Hunger disease. New York: John Wiley and Sons, 11-43.

Kirbas, D., Sutlas, N., Kuscu, D. Y., Karagoz, N., Tecer, O., \& Altun, U. (2008). The impact of prolonged hunger strike: clinical and laboratory aspects of twenty-five hunger strikers. Ideggyogy Sz., 61(9-10), 317-24.

Leiter, L. A., \& Marliss, E. B. (1982). Survival during fasting may depend on fat as well as protein stores. JAMA. 248(18), 2306-7. http://dx.doi.org/10.1001/jama.1982.03330180066037

Naghibi, M., Smith, T. R., Elia, M., (2014). A systematic review with meta-analysis of survival, quality of life and cost-effectiveness of home parenteral nutrition in patients with inoperable malignant bowel obstruction. Clin Nutr.

Oh, S. Y., Jun, H. J., Park, S. J., Park, I. K., Lim, G. J., Yu, Y., Cho, S. J., \& Song, A. (2014). A randomized phase II study to assess the effectiveness of fluid therapy or intensive nutritional support on survival in patients with advanced cancer who cannot be nourished via enteral route. J Palliat Med., 17(11), 1266-70. http://dx.doi.org/10.1089/jpm.2014.0082

Pironi, L., \& Ruggeri, E., (1997). Tanneberger S, Giordani S, Pannuti F, et al. Home artificial nutrition in advanced cancer. J R Soc Med, 90, 597-603.

Schneider, S. M., \& Hébuterne, X. (2015). Nutritional support of the elderly cancer patient: long-term nutritional support. Nutrition. 31(4), 617-8. http://dx.doi.org/10.1016/j.nut.2014.11.005

Senesse, P., Tadmouri, A., Culine, S., Dufour, P. R., Seys, P., \& Radji, A. (2015). Rotarski M, Balian A, Chambrier C. A prospective observational study assessing home parenteral nutrition in patients with gastrointestinal cancer: benefits for quality of life. J Pain Symptom Manage, 49(2), 183-191. 
http://dx.doi.org/10.1016/j.jpainsymman.2014.05.016

Vashi, P. G., Dahlk, S., Popiel, B., Lammersfeld, C. A., Ireton-Jones, C., \& Gupta, D. (2014). A longitudinal study investigating quality of life and nutritional outcomes in advanced cancer patients receiving home parenteral nutrition. BMC Cancer, 15, 14-593. http://dx.doi.org/10.1186/1471-2407-14-593

Winick, M. (1979). Hunger disease: studies by the Jewish physicians in the Warsaw Ghetto. New York: John Wiley and Sons.

\section{Copyrights}

Copyright for this article is retained by the author(s), with first publication rights granted to the journal.

This is an open-access article distributed under the terms and conditions of the Creative Commons Attribution license (http://creativecommons.org/licenses/by/4.0/). 